\title{
Erratum to: A computer model for evaluating the osteotomy parameters of Chiari pelvic osteotomy
}

\author{
Mohsen Karami • Dariush Gouran Savadkoohi •
}

Ali Ghadirpour • Sina Rahimpour •

Mahmood Azghani • Farzam Farahmand

Published online: 9 December 2009

(C) Springer-Verlag 2009

Erratum to: International Orthopaedics (SICOT)

DOI 10.1007/s00264-009-0769-2

Due to an oversight in production, one of the author's names was rendered incorrectly. The correct spelling is:

Sina Rahimpour

The online version of the original article can be found at http://dx.doi. org/10.1007/s00264-009-0769-2.

M. Karami $(\bowtie)$

Department of Orthopaedics,

Shahid Beheshti University of Medical Sciences,

Tehran, Iran

e-mail: mn.karami@gmail.com

D. G. Savadkoohi $\cdot$ A. Ghadirpour $\cdot$ S. Rahimpour

Sina Trauma Research Center,

Tehran University of Medical Sciences,

Tehran, Iran

M. Azghani - F. Farahmand

Sharif University of Technology,

Tehran, Iran 